\title{
Brésil : les nouvelles faces de la violence
}

\section{Angelina Peralva}

\section{(2) OpenEdition}

\section{Journals}

\section{Édition électronique}

URL : http://journals.openedition.org/conflits/676

DOI : $10.4000 /$ conflits.676

ISSN : $1777-5345$

Éditeur :

CCLS - Centre d'études sur les conflits lilberté et sécurité, L'Harmattan

\section{Édition imprimée}

Date de publication : 16 juillet 1992

ISSN : 1157-996X

\section{Référence électronique}

Angelina Peralva, «Brésil : les nouvelles faces de la violence », Cultures \& Conflits [En ligne], 06 | été 1992, mis en ligne le 06 janvier 2003, consulté le 30 mars 2021. URL : http://journals.openedition.org/ conflits/676 ; DOI : https://doi.org/10.4000/conflits.676

Ce document a été généré automatiquement le 30 mars 2021.

Creative Commons License 


\title{
Brésil : les nouvelles faces de la violence
}

\author{
Angelina Peralva
}

La généralisation de la violence comme problème

1 La question de la violence n'est certes pas nouvelle au Brésil. Mais sa montée en force, les formes étonnantes qu'elle prend en ce début de décennie, semblent plus en rupture qu'en continuité avec un passé récent. Elle devient, au sens propre, un mode de régulation des interactions humaines et cela est nouveau. A Touraine, il n'y a pas longtemps, avait parlé d'un clivage, central selon lui pour l'ensemble du continent latino-américain, entre le monde de la parole, le monde de la modernité, et le monde du sang, celui des laissés-pour-compte ${ }^{1}$. Aujourd'hui il n'y a plus de monde de la parole.

2 Le monde des in était un monde régulé, soumis à des normes. Des dérégulations partielles, bien sûr, ont toujours existé. Mais aujourd'hui, il n'y a plus de règles.

3 En vrac, quelques indications. La première dérégulation, et la plus importante sans doute, est celle qui renvoie au brouillage, à une échelle jamais vue auparavant, des frontières entre le public et le privé. Autrefois on dénonçait la corruption pratiquée à de hauts échelons de l'administration publique, comme une transgression. Aujourd'hui la corruption s'exerce verticalement à tous les échelons de l'administration, depuis les plus hauts, jusqu'aux plus bas et les plus démunis de ressources.

4 Autrefois on essayait d'imposer des limites au trafic et à la consommation des drogues. Aujourd'hui le trafic de drogues dures se fait de plus en plus ouvertement sur des lieux publics. Dans le courant de l'année 1991, des dispositions ont dû être prises pour empêcher la libre circulation des trafiquants dans l'enceinte du Congrès national et, à l'université de Sao Paulo, les lieux du rectorat ont fait l'objet de restrictions analogues.

Il n'y a pas longtemps, la police devait faire face à des enlèvements de banquiers et grands patrons, pratiqués par des professionnels du crime. Aujourd'hui, de plus en plus souvent, des amateurs s'y mettent, sans aucun souci du choix de la victime. N'importe qui peut se faire enlever, contre des sommes dérisoires et on a même introduit une formule nouvelle : la menace d'enlèvement, où l'enlèvement même est suspendu au cas où la rançon serait préalablement payée. 
6 La contrepartie: une prolifération des sociétés de vigiles, chargées de la sécurité privée $^{2}$ et la généralisation du port d'armes. Quelques faits divers, au cours du second semestre 1991 : un couple réagit à un supposé hold-up dans le centre-ville de Sao Paulo, en tuant d'une balle dans la tête le jeune qui les avait approchés; un retraité, dans un quartier populaire de Sao Paulo, tue d'une balle dans la tête un graffiteur de 14 ans et se suicide après ; un client tue, dans le parking d'un supermarché, un jeune cycliste ayant collé involontairement à sa voiture.

7 L'accroissement du rythme des lynchages est spectaculaire. Pour le seul État de Bahia on signale 70 épisodes au cours du premier semestre $1991^{3}$. Ces événements pourtant ne peuvent pas être interprétés comme l'expression d'une violence de source populaire. Certains chercheurs proposent de distinguer le lynchage anomique, suscité à partir de certains incidents et pas toujours achevé; et le lynchage communautaire, qui correspond à une action collective plus ou moins délibérée ${ }^{4}$. Dans les deux cas, des personnes de toutes origines sociales peuvent être impliquées.

8 En 1982, un procureur de la République a tué en plein centre-ville de Sao Paulo, en le foulant sous ses pieds devant le regard des passants, un gamin des rues qui avait arraché à une dame une petite chaîne en or. En 1990, le lynchage de Matupa, petit village du Mato Grosso, filmé en vidéo par un cinéaste amateur, est devenu célèbre par ses images, diffusées dans le monde entier. Deux conseillers municipaux, un commerçant et sept policiers sont accusés de participation à cet événement. La violence de la police est ancienne ; mais elle apparaissait jusqu'ici soit comme l'expression d'un État autoritaire, soit comme débordement. Aujourd'hui il n'y a plus de digues, parce qu'il n'y a plus d'État. Encore un fait divers pour l'illustrer: en 1991, un incendie d'origine criminelle a fait vingt victimes parmi les prisonniers de la Maison d'arrêt Ary Franco à Rio de Janeiro. L'enquête a rapidement établi la responsabilité directe d'un chef maton, ayant voulu se venger d'un détenu.

9 A défaut d'État, comme dans une sorte de succédané spasmodique, la société civile et ses juristes s'engagent dans un débat sur l'instauration de la peine de mort et l'imputabilité du mineur dès l'âge de douze ans 5 .

Le pauvre, victime de la violence

10 Le premier aspect à prendre en considération dans la violence des pauvres, c'est le fait qu'ils en sont souvent les principales victimes. Certains faits sont anciens. Précairement logés, ils sont les proies faciles des cataclysmes naturels, comme les pluies torrentielles qui, chaque été, dans de grandes villes telles Sao Paulo et Rio de Janeiro, accroissent le nombre de ceux qui logent dans la rue. S'il n'y a pas d'intentionnalité directe de destruction de l'autre dans ces phénomènes, la violence est ici reconnue comme le produit naturel d'une société qui admet l'exclusion, y compris jusqu'à la limite où le droit même à la vie est nié.

11 D'autres faits sont plus récents, comme l'extermination d'enfants et d'adolescents pauvres, qui occupe une place spéciale dans le débat actuel. Tout d'abord, en raison de l'extension prise par ce phénomène. La presse signale 918 meurtres d'enfants et adolescents à Sao Paulo, en 1990, dont $30 \%$ attribués à la police.

12 En 1970, en plein régime militaire, le nombre total des meurtres attribués aux escadrons de la mort aurait été de l'ordre de 220 presque tous ayant comme cible des adultes $^{6}$. La ville de Recife présente le plus fort taux de meurtres d'enfants par tête habitant, suivie de Rio de .Janeiro et Sao Paulo 7 . Les motivations qui se cachent derrière 
ce phénomène d'extermination sont mal connues. Témoins permanents de tout ce qui se passe dans la rue, les enfants seraient éliminés pour en savoir trop sur le monde du crime - voilà une des explications le plus souvent avancées 8 . Il est pourtant difficile de croire que ces enfants puissent représenter un vrai risque pour les délinquants. Une étude portant sur les villes de Sao Paulo, Rio de Janeiro et Recife, malgré son caractère exploratoire et le fait de s'appuyer sur des sources secondaires, propose des indications intéressantes que nous résumerons ici, car elles permettent d'asseoir quelques hypothèses.

13 1) D'après les informations disponibles, concernant la période mars/août 1991, 72 \% des enfants assassinés n'avaient jamais figuré dans des fichiers de la police; $68 \%$ ne portaient pas d'armes au moment du crime; $74 \%$ n'avaient jamais été accueillis dans des institutions officielles. II faut pourtant noter que, pour la plupart des meurtres considérés dans l'étude, ces informations n'étaient pas disponibles.

2) On sait, par ailleurs, que seuls $2 \%$ des enfants et des adolescents assassinés portaient ou présentaient des signes d'ingestion de drogues (11 parmi 457 meurtres analysés); dans $8 \%$ des cas (38 parmi 457 meurtres), il y avait des liens évidents entre ces enfants et des narcotrafiquants. Ce constat suggère la relativisation d'une autre "explication" de l'extermination: celle qui prétend que, pris dans le réseau de distribution de la drogue, ces enfants seraient assassinés pour ne pas avoir respecté les règles du jeu.

3) L'étude montre encore que dans $88 \%$ des cas sur lesquels on dispose d'informations (252 sur 286), ces enfants avaient un domicile fixe.

4) Constat plus intéressant : parmi 56 auteurs de meurtres identifiés, pour lesquels des informations étaient disponibles, 37,5\% n'avaient aucun lien avec l'enfant ; 37,5 \% par contre étaient des membres de sa famille et $18 \%$ avaient avec lui des liens de voisinage 9

17 Ces informations permettent d'avancer l'hypothèse que l'extermination d'enfants et adolescents au Brésil combine aujourd'hui de manière perverse, trois ordres de problèmes, qu'il est important de distinguer. Le premier relève de la crise économique et sociale. Tous les indicateurs - accroissement du chômage, déscolarisation, déficit de logements - opèrent dans le sens d'une aggravation des situations d'exclusion. Celles-ci favorisent par contre un phénomène très large d'occupation des rues des grandes villes par des bandes d'enfants, qui constituent autant de victimes probables de tous les risques encourus lors d'une utilisation non régulée de l'espace urbain. Une enquête réalisée par des chercheurs de l'université catholique de Sao Paulo auprès de l'Institut médico-légal de la région du Grand $\mathrm{ABCD}^{10}$, a permis de constater que le renversement par des voitures et les noyades figuraient parmi les principales causes de décès des enfants des rues.

Deuxièmement, en ce qui concerne les meurtres, le lien, observé dans le bilan précité, entre l'auteur et sa victime, est suffisamment significatif pour justifier l'hypothèse d'une simple banalisation de la violence, face à la décomposition de formes alternatives de régulation des interactions humaines. Certaines enquêtes ont montré la nature très fortement endogène de la violence dont fait l'objet la population pauvre - les relations compliquées entre travailleurs et délinquants, les modes de démarcation de territoires, au départ invisibles au regard étranger ${ }^{11}$.

19 Le troisième problème concerne l'implication de la police, et donc la responsabilité de l'État, dans ces meurtres, avec deux faces. D'un côté, celle de l'extermination délibérée, 
toujours plausible, dans la mesure où des antécédents historiques existent ${ }^{12}$. L'autre face, plus vraisemblable, exprime non pas la violence d'un État autoritaire, mais sa décomposition. Aussi, le constat, maintes fois renouvelé, d'une participation de la police militaire et de la police civile dans les assassinats d'enfants doit suggérer une question simple : au moment du crime, celui qui le commet le fait, investi dans son rôle de fonctionnaire de l'État, ou, au contraire, dans celui d'un individu quelconque, immergé dans le même cadre de banalisation de la violence qui touche l'ensemble de la population?

Le pauvre, acteur de la violence

Des voix, à juste titre, s'insurgent aujourd'hui contre une équation récurrente, postulant que la criminalité dérive de la pauvreté et qu'elle est donc porteuse d'un sens de révolte sociale. Frise, au contraire, entre les mailles des professionnels du crime, l'action des jeunes délinquants serait purement hétéronome, les emmenant très vite à la prison ou à la mort ${ }^{13}$.

21 Ces observations sont d'autant plus justes et importantes, dans la mesure où nous vivons un moment où la généralisation de la violence brouille les cartes, à un tel point qu'il devient difficile de dire si la révolte est partout ou si elle n'est nulle part. Néanmoins, et malgré cet impératif éthique, il est vrai aussi qu'il faut interroger le sens caché derrière le langage de la violence. De ce point de vue, réduire l'action des jeunes délinquants à la pure hétéronomie reste insuffisant.

Lors de ses recherches du début des années 80 , menées dans un ensemble HLM de la banlieue de Rio, A. Zaluar avait pu distinguer, dans l'univers de la pauvreté, deux logiques, définissant des modèles de comportement divers pour les groupes qui s'y référaient. D'un côté le travailleur, qui, dans une ville de services comme Rio de Janeiro, a souvent une qualification imprécise, semblait structurer son identité autour de l'idée qu'il y a toujours du travail, donc travailler c'était toujours possible; et il cherchait à jouer un rôle de pourvoyeur du groupe familial. Le jeune, au contraire, aspiré par la société de consommation, exprimait souvent des sentiments négatifs par rapport au travail et il pouvait, même si cela n'arrivait pas toujours, dériver vers la délinquance, si celle-ci était perçue par lui comme voie rapide d'accès à la richesse. Dans l'univers du travail, le travailleur s'opposait au patron; dans l'univers de la consommation, le pauvre s'opposait au riche. Certaines formes actuelles de violence urbaine semblent en effet dériver de chacune de ces deux logiques. Déjà en 1983, lors d'une vague d'attaques contre des supermarchés dans les quartiers populaires de Rio, Zaluar constatait que ces actions associaient à la fois des travailleurs et des délinquants, mais avec des agendas différents : les travailleurs se procuraient de la nourriture, les délinquants des produits de luxe.

Depuis deux ans, des affrontements meurtriers ont régulièrement opposé des ambulants et des commerçants dans les rues du centre ville de Sao Paulo et Rio de Janeiro. Ces événements relèvent, bien entendu, d'une dérégulation des espaces de travail. Ils appartiennent au même registre que les casses émeutières dans les transports en commun, ayant comme cible des autobus autant que des trains de banlieue". Par contre, les "dépouilles" de baskets dont les victimes sont souvent des enfants des couches moyennes, et qui ont été à l'origine, en 1991 à Sao Paulo, de trois meurtres en l'espace de trois mois, se situent évidemment dans le domaine de la consommation symbolique des produits grandes marques. Aussi une nouvelle forme de "dépouille" s'est emparée des plages de Rio, l'arrastao - expression utilisée par analogie 
avec le traîneau du pêcheur. Sa signification expressive semble au moins aussi importante que sa portée instrumentale. Des bandes d'enfants et d'adolescents par dizaines, armés de bâtons, avec des clous au bout, descendent des bidonvilles plantés au beau milieu des quartiers riches et parcourent toute l'extension de la plage, ramassant ce qu'ils rencontrent: argent, montres, baladeurs, des fois caleçons et maillots de bain... Ce phénomène, relativement nouveau, a modifié la dynamique, sur la plage, des vols à la tire, où le voleur se devait surtout d'être malin; mais il a modifié, au-delà la dynamique même du vol à main armée, où l'affrontement opposait en face à face le voleur et sa victime. C'est peut-être la généralisation croissante des phénomènes de lynchage anomique pour employer l'expression de Benevides, qui pousse en contrepartie à la formation de cette caricature d'acteur collectif Contrairement à l'image classique du voleur, dans l'arrastao on ne se cache pas, on ne se veut pas malin, mais on affirme plutôt la force du groupe. La logique du travail et celle de la consommation n'épuisent pourtant pas à elles seules les significations à l'oeuvre dans ces nouvelles formes de violence urbaine. Quoi de plus violent, mais aussi quoi de plus purement expressif que le "surf", pratiqué par des jeunes pauvres sur les trains de banlieue de Rio, ville où le surf - le véritable, celui qui se pratique en mer - est l'apanage des jeunes des quartiers riches? Le rapport aux médias et l'accès du sujet à l'espace public, dans un cercle infernal d'affirmation de soi et d'autodestruction, semblent des éléments de motivation importants qui se cachent derrière ce type de conduite.

Tant de violence, pourquoi?

La généralisation de la violence comme mode de régulation des interactions humaines suppose la multiplication des formes à travers lesquelles elle se manifeste. Il ne s'agit pas ici d'épuiser la description de ces formes, qui par ailleurs évoluent constamment et parfois disparaissent, avant même de se fixer en tant que types. Lorsqu'on s'interroge, par contre - et il est indispensable de le faire - sur les raisons d'une telle accélération de la dynamique de la violence dans l'univers urbain brésilien, la réponse n'est sûrement pas aisée. Malgré une abondante littérature consacrée à ce thème, les recherches actuelles ont du mal à rendre compte du phénomène dans son ensemble. On manque de catégories pour analyser le chaos.

Une interprétation déjà ancienne consiste à expliquer le phénomène de la violence du point de vue des rapports entre le monde de la modernité et celui des exclus. C'est d'une certaine manière l'hypothèse de La parole et le sang, proposée par A Touraine. C'est aussi l'hypothèse de Roberto da Matta, pour qui les exclus du monde de la parole ne peuvent y accéder que par la violence, même sous des formes symboliques, qu'il résume dans une expression bien connue de chaque Brésilien: "Savez-vous à qui vous vous adressez ?" ${ }^{14}$. C'est encore un peu, il me semble, la démarche plus récente de Gilberto Velho, qui s'intéresse au mode de constitution et aux rapports réciproques entre in et out-groups ${ }^{15}$.

Ces hypothèses s'inscrivent toutes plus ou moins dans la perception générale d'une société où le développement s'est fait aux dépens d'une capacité d'intégration au demeurant très faible. La violence des exclus, ainsi que celle déployée à leur égard, apparaissait alors comme le pendant d'une modernité ne bénéficiant que d'un nombre d'élus limité. Cependant, lorsque la violence se généralise, comme à l'heure actuelle, la pertinence de ces catégories semble cesser. La violence n'est plus un langage inversé, par lequel s'opérerait la communication entre deux groupes distincts d'acteurs, elle devient l'unique langage par lequel l'ensemble de la société s'exprime. 

des effets importants du point de vue d'une dégradation générale des conditions de vie. On sait aujourd'hui par exemple, que l'apport de l'enfant représente une part considérable des revenus familiaux, ce qui le pousse à quitter vite l'école ou à la fréquenter irrégulièrement; ce qui le pousse aussi, pris dans la dynamique des petits boulots qu'il entreprend, à ne pas rentrer chez lui pendant plusieurs jours; ce qui entraîne, enfin, un affaiblissement radical de la famille en tant que lieu de socialisation, favorisant le déploiement de la violence sous les formes les plus diverses ${ }^{16}$. Mais peuton tout expliquer par la crise économique?

Dès les années 1980, la question de la violence avait pénétré le débat universitaire, à travers l'idée d'une démocratisation nécessaire de l'État, capable de le rendre garant des droits du citoyen. Ce versant a défini tout un ensemble d'études ayant comme objet les institutions répressives: la police civile ${ }^{17}$, la gendarmerie ${ }^{18}$, les prisons ${ }^{19}$. D'une manière générale, ces recherches ont jusqu'ici montré que plutôt que d'opérer comme un élément de limitation de la violence au sein de la société civile, l'État brésilien au contraire opérait dans le sens de son exacerbation.

Actuellement, l'une des hypothèses fortes qui orientent cet ensemble de recherches se construit autour de la notion d'"autoritarisme socialement implanté". Dans cette perspective, la violence est comprise comme le sous-produit d'une culture politique propre à la société brésilienne, prête à soutenir des régimes autoritaires et assurer leur succès ${ }^{20}$.

Et pourtant, peut-on aujourd'hui encore parler à juste titre d'autoritarisme, et qui plus est, socialement implanté, alors que l'État se délite et qu'au sens propre il n'est plus ? Si on veut partir de l'État, il faut interroger sa crise. A cet égard, les années 1980 sont un moment charnière. Deux thèmes condensent les efforts déployés dans le pays en cette décennie-là : celui de la démocratisation institutionnelle et celui de la dette extérieure.

Sortant d'une longue dictature, pendant un moment, pour les Brésiliens, tout l'avenir semblait se jouer autour de l'Assemblée constituante qui en 1987 a promulgué une nouvelle charte. Au cours des longs mois qui ont précédé ces définitions, le pays s'est converti en un immense groupe de pression - chaque petit serment de la société voulant faire peser son point de vue particulier sur la loi fondamentale. La dette extérieure, d'autre part, saignait le pays au moment même où le flux des capitaux, ayant bénéficié l'Amérique latine au cours de la décennie précédente, s'était inversé.

Terrible paradoxe de ces années-là : alors que pour l'ensemble des acteurs politiques tout semblait se jouer sur le plan institutionnel, dans le domaine économique l'État se vidait de tout pouvoir. L'épuisement du débat institutionnel n'a pas tardé. Le dernier affrontement politique national date des élections présidentielles de 1989. Depuis, c'est le néant.

Un feuilleton brésilien

Dans la suite de cet effort d'explication du phénomène de généralisation de la violence, à l'oeuvre à l'heure actuelle, les élections de 1989 recèlent un événement fondateur, qui nous renvoie à une dernière hypothèse, à la Durkheim, celle de la crise morale profonde qui ronge la société brésilienne.

34 Le projet battu, celui du candidat du Parti des travailleurs, exprimait une tentative d'articulation de trois logiques distinctes : il parlait au nom des exclus, au nom de leur intégration à la modernité ; au nom des travailleurs et d'une sensibilité de type syndical 
' et finalement au nom d'une sensibilité in, celle des couches moyennes modernes, soucieuses d'un développement assorti d'une plus forte capacité d'intégration sociale. Il proposait, dans la foulée, la cessation des paiements de la dette extérieure, faisant appel à un développement national autocentré.

Le projet vainqueur, celui de l'actuel président Fernando Collor de Mello, parlait aussi au nom des exclus et de la modernité ; mais en faisant surtout confiance à une plus forte intégration du pays au marché international, et en prônant par conséquent le paiement intégral de la dette.

Au-delà, donc, du public auquel ces deux candidats s'adressaient, leur message était empreint d'une mise en valeur de logiques différentes: d'un côté, la solidarité des travailleurs et celle de la nation; de l'autre le langage du libéralisme et du marché. Les élections se sont terminées sur un fond de violence, à l'américaine. Payée par les organisateurs de la campagne Collor, une ex-femme du candidat du Parti des travailleurs a déclaré à la télévision que ce dernier lui avait demandé d'avorter, lorsque quinze ans auparavant elle s'était retrouvée enceinte de lui. Lula s'est tu; sa fille, fortement traumatisée, dut être envoyée en clinique de repos; et son adversaire a gagné les élections - grâce à la mise à mort publique, consentie par le pays, d'une enfant de quinze ans.

A suivre...

\section{NOTES}

1. La parole et le sang, Paris, Ed. Odyle-Jacob, 1988.

2. Voir à ce propos les indications de A L. Paixao, "Segurança privada, direitos humanos e democracia. Nolas preliminares subre nouos dilemas politicos", Novos Estudos CEBRAP (31), outubro de 1991, pp. 131-141.

3. Folha de Sao Paulo, 18 de julho de 1991. Les lynchages sont un phénomène ancien au Brésil. Néanmoins, et à défaut de statistiques plus précises, des enquêtes ont pu partir de la presse à grande diffusion. Entre septembre 1979 et janvier 1982, on signale 32 épisodes de lynchage avec des morts et 43 tentatives de lynchage. Ce chiffre, correspondant à une période de 28 mois, est donc l'équivalent de celui qu'on enregistre pour le seul État de Bahia, au cours du premier semestre 1991. Cf. M. V. Benevides, "Linchamentos : violência e justiça popular" in divers auteurs,Violência Brasileira., SP Brasiliense, 1982.

4. Cf. M. V. Benevides, op. cit.

5. A ce propos, cf W. Barreira, "Violência cresce nas grandes ciudades enquanto a a çao contra a delinquência juvenil divide juristas", O Estado de S. Paulo, 9 de novembro de 1991.

6. Marques Joao Benedicto de Azevevo, "Autoridades precisam esclarecer execuçoes sumarias", O Estado de S. Paulo 9 de novembro de 1991.

7. Cf. MNMMR, IBASE, NEV, USP, Vidas em risco. Assassinatos de crianças e adolescentes no Brasil. RJ 1991. 
8. Dimenstein Gilberto, A guerra dos meninos. Assassinatos de menores no Brasil, SP, Brasiliense, 1991.

9. MNMMR, IBASE, NEV, USP, Vidas em risco, op. cit.

10. Il s'agit des villes de Santo André Sao Bernardo, Sao Caetano et Diadema l'un des plus importants pôles industriels brésiliens. Les résultats de l'enquête de la PUC-SP ne sont pas encore publiés.

11. Cf. par exemple l'enquête du journaliste Percival de Souza, A maior violencia do mundo, Baixada Fluminense, Rio de Janeiro, Brasil. Sp, Traço Editora, 1980. Et aussi l'ex ?llrnt travail dAlba Zahuar, A maquina e a revolta, As organizaçoes populares e o significado da pobreza SP, Brasiliense, 1985.

12. Dans les années 60, le directeur du Département d'ordre politique et social, un organe de la polire civile, prit la décision de délivrer la ville de Rio de Janeiro de ses clochards. Les corps ont flotté dans une rivière des environs, le Rio da Guarda.

13. Cf. A. Zaluar, "Tristes desgraças do libertarismo", Jornal do Brasil, 4 VIII-1991.

14. R. da Matla, Carnarvais, mlandras e herois. RJ, Zahar, 1979, "As raizes da violência no Brasil : reflexoes de um antropologo social", in Divers auteurs, Violência brasileira. SP Brasiliense, 1982.

15. G. Delho, "O grupo e seus limites", Revista da USP (9), março/abril/maio de 1991.

16. Sur ces thèmes, cf. particulièrernent "Sobrevivrr em Sao Paulo" e "Ter medo em Sao Paulo", in V C. Brant (c ?ord.), Sao Paulo : Trabalhar e viver, sp, Comissao de Juatiça e Paz/Editora Brasiliense, 1982. C( aussi, J. S. Martins (coord.), O massacre dos inocentes, A criança sem infância no Brasil, SP, Hucitec, 1991.

17. R. M. Fischer. O Direito da Populaçao à Segurança. Cidadania e violência urbana, Petropolis, CEDEC/Vozes, 1985.

18. P. S. Pinheiro, "Policia e crise politica : o caso das policias militarea", in Divers auteurs : Violência brasileira. Sao Paulo, Brasiliense, 1982. P S. Pinheiro et alii, "Violência Fatal", Revista da USP (9), março/abril/maio 1991.

19. P. S. Pinheiro (org). Crime, violência e poder, SP Brasiliense, 1983. M. M. P Castro, "Ciranda do medo", Revista da USP (9), março/abril/maio 1991. S. Adorno, "Sistema penitenciario no Brasil", Revista da USP (9), maço/abril/maio 1991.

20. P S. Pinheiro, "Autoritarismo e transiçao", Revista da USP (9), março/abril/maio 1991. E. Dellassopa, "Reflexoes sobre a violência, autorridade e autoritarismo", Revista da USP (9), março/abril/maio de 1991.

\section{RÉSUMÉS}

Autrefois langage inversé, créant un lien entre deux mondes, celui de la modernité et celui des exclus, la violence devient aujourd'hui, dans le Brésil urbain; l'unique langage à travers lequel la société s'exprime. II y a donc rupture, plus que continuité par rapport à un passé récent. L'article examine les nouvelles formes que prend cette violence et s'interroge sur les différentes hypothèses susceptibles d'expliquer sa généralisation en tant que mode de régulation des interactions humaines. 
In former days, violence was the anti-language between two worlds : the modern world and the world of the excluded. In today's Brazilian cities, violence is the only language of society. There is thus a break, rather than continuity, with the recent past. The article examines the new forms of violence and studies different hypotheses explaining the generalisation of this phenomenon as a means of regulating human interaction.

INDEX

Mots-clés : violence, pauvreté

Index géographique : Amérique du Sud, Brésil

\section{AUTEUR}

\section{ANGELINA PERALVA}

Professeur à l'Université de Sao Paulo, et chercheur au Centre d'Analyse et d'Intervention Sociologiques. 